\title{
Erratum to: Biochemistry of primary headaches: role of tyrosine and tryptophan metabolism
}

\author{
G. D'Andrea $^{1} \cdot$ S. Cevoli ${ }^{2}$ D. Colavito ${ }^{1} \cdot$ A. Leon ${ }^{1}$
}

Published online: 24 December 2015

(C) Springer-Verlag Italia 2015

\section{Erratum to: Neurol Sci (2015) 36 (Suppl 1):S17-S22}

DOI 10.1007/s10072-015-2131-3

The author would like to correct the affiliation details of the co-author Prof. Sabina Cevoli.

The correct affiliation is given below:

S. Cevoli

Neurology Department, Headache Center,

IRCCS Institute of Neurological Sciences, Bologna, Italy.

The online version of the original article can be found under doi:10.1007/s10072-015-2131-3.

D. Colavito

colavito@ researchinnovation.com

1 Research \& Innovation (R\&I), Padua, Italy

2 Neurology Department, Headache Center,

IRCCS Institute of Neurological Sciences, Bologna, Italy 